\title{
Therapeutic Comparison between Sun Irradiation vs. Narrowband UVB Phototherapy along with Concomitant Topical Tacalcitol for Vitiligo Vulgaris"
}

\author{
Atsushi Tanemura ${ }^{1 \#}$, Aya Takahashi ${ }^{1}$, Yuriko Ueki ${ }^{1}$, Hiroyuki Murota ${ }^{1}$, Yuji Yamaguchi ${ }^{2}$, \\ Ichiro Katayama ${ }^{1}$ \\ ${ }^{1}$ Department of Dermatology, Graduate School of Medicine, Osaka University, Osaka, Japan; ${ }^{2}$ Department of Geriatric and Environ- \\ mental Dermatology, Nagoya City University Graduate School of Medical Sciences, Nagoya, Japan. \\ Email: "tanemura@derma.med.osaka-u.ac.jp
}

Received January $27^{\text {th }}, 2012$; revised February $28^{\text {th }}, 2012$; accepted March $9^{\text {th }}, 2012$

\begin{abstract}
In addition to therapeutic options such as steroid ointment, immunosuppressive drug and ultraviolet phototherapy, recent reports have demonstrated the clinical efficacy of topical vitamin D3 for vitiligo vulgaris. However, there seems to be little clinical effect of vitamin D3 without UV exposure. In the present study, we evaluated the clinical difference of sun irradiation vs. narrowband UVB in combination with tacalcitol assessing the change of lesion size and color tone by a spectrophotometer. Thirty-three vitiligo patients were composed of 19 treated with sun illumination and 14 treated with narrowband UVB in combination with topical use of tacalcitol. The mean \% size reduction was higher in the group of sunbathing than narrowband UVB (29\% vs. 23\%). Delta L score, which represented the difference of whiteness between lesional and perilesional skin, was significantly improved after treatment in the group of sun irradiation instead of narrowband UVB $(\mathrm{p}=0.0023)$. Therefore, we consider that sun illumination along with tacalcitol may be able to induce natural repigmentation and be an alternative therapeutic option for vitiligo vulgaris.
\end{abstract}

Keywords: Vitiligo; Tacalcitol; $\left[1 \alpha 24(\mathrm{OH})_{2} \mathrm{D}_{3}\right]$; Sun Irradiation; Narrowband UVB

\section{Brief Report}

Despite treatment of vitiligo vulgaris by steroids, phototherapy, and epidermal grafts, especially for segmental type, no standard regimen has yet been established to overcome vitiligo [1]. Activated vitamin $\mathrm{D}, 1 \alpha 24(\mathrm{OH})_{2} \mathrm{D}_{3}$, is an alternative treatment for vitiligo that accelerates repigmentation through biological interaction between keratinocytes and/or fibroblast to melanocytes [2]. Narrowband UVB is commonly accepted because it provides more effective repigmentation and is less carcinogenic than broadband UVB and calcipotriol along with phototherapy is also very promising $[3,4]$. On the other hand, solar irradiation following topical use of tacalcitol induces natural repigmentation through the upregulation of c-Kit [5]. This study assesses the change of lesion size and color tone after treatment of narrowband UVB or by sunbathing along with the topical use of tacalcitol for vitiligo.

Thirty-three vitiligo patients under treatment at the department of dermatology in Osaka University Hospital

${ }^{*}$ Conflict of Interest: none.

${ }^{*}$ Corresponding author. were enrolled after they provided their written informed consent and a total 41 vitiligo lesions were assessed during the spring to autumn season. The detailed demographics of the enrolled patients are shown in Table 1. The patients were randomly divided into 2 treatment groups composed of solar illumination and weekly narrowband UVB therapy in combination with topical use of tacalcitol $\left[1 \alpha 24(\mathrm{OH})_{2} \mathrm{D}_{3}\right]$ once dairy in the morning. We assured no significant difference of clinical background including age, gender, disease duration and lesion sites in each group by chi-squared test. The patients were excluded if corresponding to the following exclusion criteria: presence of pretreatment within 1 month, contact dermatitis by topical vitamin D3 analogue, skin type I or II, segmental vitiligo. Nineteen patients were instructed to sunbathe for 30 minutes during 10 am to 14 pm within 1 hour after topical application of tacalcitol to the affected lesions every day (Group A). Fourteen patients received narrowband UVB311nm phototherapy once or twice a week, with a cumulative dose ranging from 7.9 to $25.8 \mathrm{~J} / \mathrm{cm}^{2}$ (mean $14.2 \mathrm{~J} / \mathrm{cm}^{2}$, Group B). The patients were given a medical interview regarding adverse events 
and other treatments during the study period monthly. The patients in both groups were instructed to apply an UV-cut cream on the uninvolved area for prevention of hyperpigmentation. The target lesions for assessment were assigned on sun-exposed area on each group and the change of lesion size and color tone using a spectrophotometer for objective color assessment (CD-2600d, Konica Minorta, Japan) was evaluated 6 months after the initial treatment. The unpaired $t$-test was used for the analysis of differences of lesion size and color tone before and after treatment and the chi-squared test was used for comparison of clinical background in each group. A value of $\mathrm{p}<0.05$ (two-tailed) was considered significant. All statistical analyses were performed using the Prism software program, version 5 (GraphPad Software Inc., La Jolla, CA, USA).

The mean single dose of narrowband UVB was 520 $\mathrm{mJ} / \mathrm{cm}^{2}$ and the cumulative dose until initial pigmentation ranged from 4.2 to $8.9 \mathrm{~J} / \mathrm{cm}^{2}$. An initial pigmentation after mean of 12 weeks and 10 weeks appeared in group $\mathrm{A}$ and $\mathrm{B}$, respectively if induced repigmentation was ob- served. The $\%$ mean repigmentation was 29 and 23 in group A and B (Figure 1(a)), respectively. Fair to excellent treatment efficacy with more than $50 \%$ repigmentation was obtained in $3(13.6 \%)$ and $4(16 \%)$ patients treated in each group. In contrast, 16 cases (34\%) showed almost no recovery at the end of this study (Figure 1(b)). A significant improvement in the delta L score, which represents the contrast of whiteness between lesion and perilesion, was observed with sun irradiation but not narrowband UVB along with tacalcitol (Figure 1(c)). Then a score, which represents the erythematous change of lesions, slightly increased only in group A not significant. The $b$ score slightly decreased in both groups, indicating the saturation decrease after treatment.

\section{Discussion}

This is the 1st study to compare the clinical effect of UV phototherapy between solar irradiation and narrowband UVB in combination with a vitamin D3 analogue for vitiligo. The results showed sunbathing to be as effective as narrowband UVB regarding the repigmentation rate,

Table 1. Clinical demographics of enrolled vitiligo patients.

\begin{tabular}{lcc}
\hline \multicolumn{1}{c}{ Clinical parameter } & Group A & Group B \\
\hline Patient/lesion number & $19 / 20$ & $14 / 21$ \\
Gender (F/M) & $13 / 6$ & $5 / 9$ \\
Age (mean) & 53.2 & 51.6 \\
Lesion site (Acrofacial/non-acrofacial) & $17 / 3$ & $16 / 5$ \\
Pretreatment history (n = yes/no) & $6 / 13$ & $6 / 8$ \\
Duration before consultation & $0-23$ yrs (mean 5.6 yrs) & $0-50$ yrs (mean 10.5 yrs) \\
Repigmentation pattern (F/P/D) $^{*}$ & $5 / 6 / 2$ & $6 / 1 / 1$ \\
\hline
\end{tabular}

F: follicular; P: peripheral; D: diffuse type.

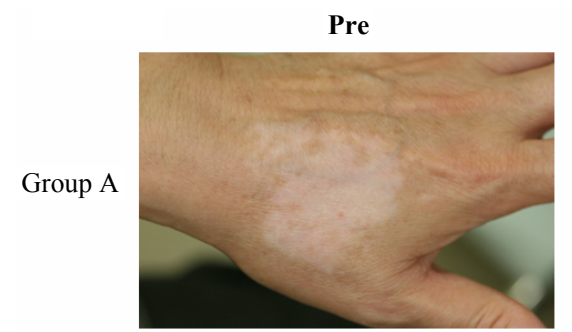

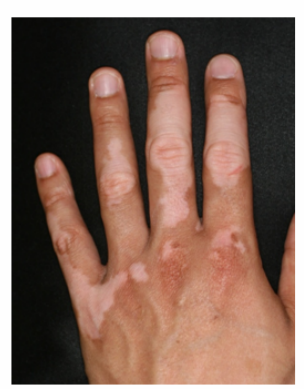

(a)

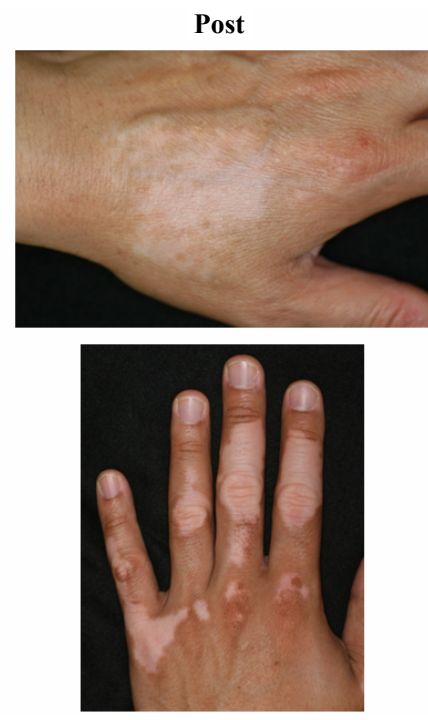




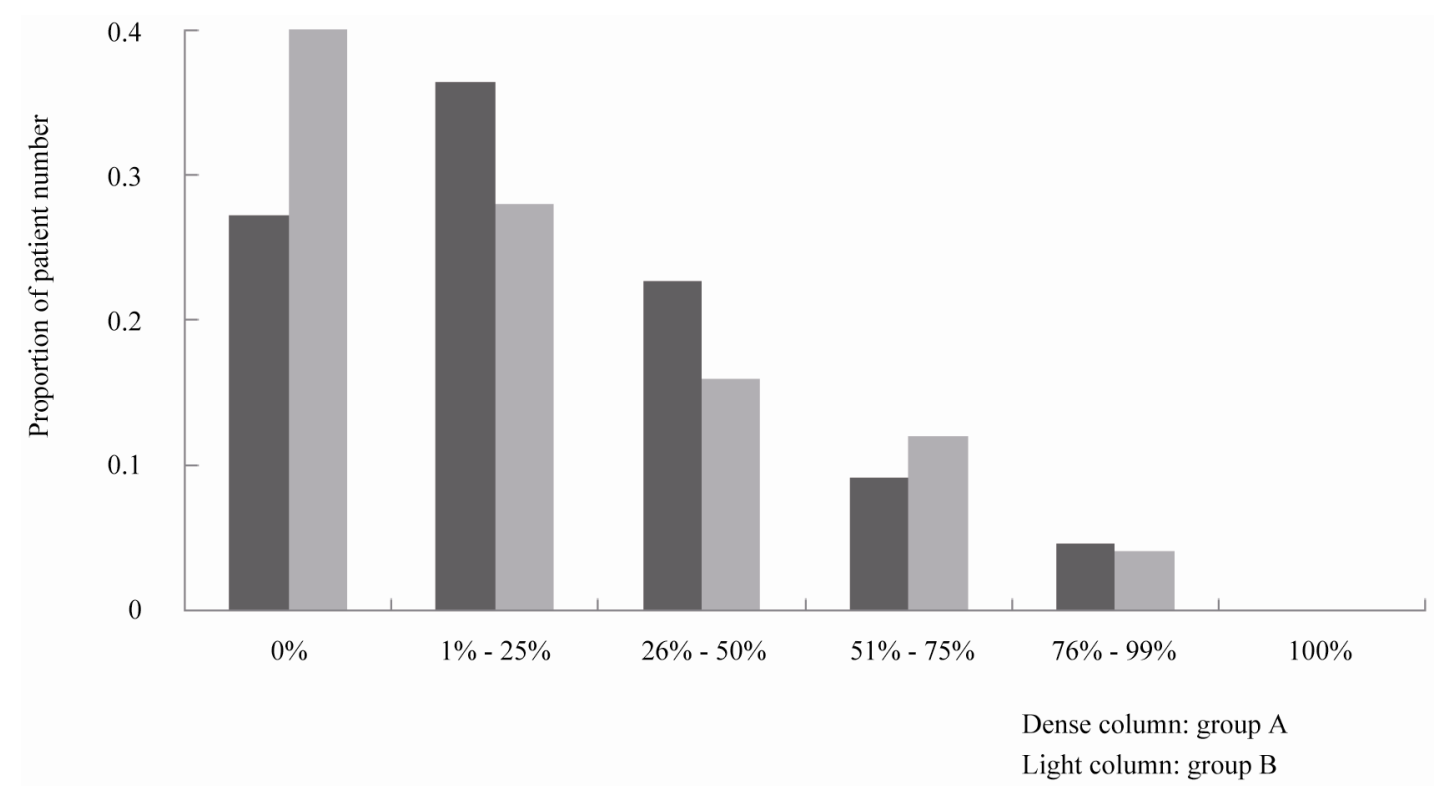

(b)
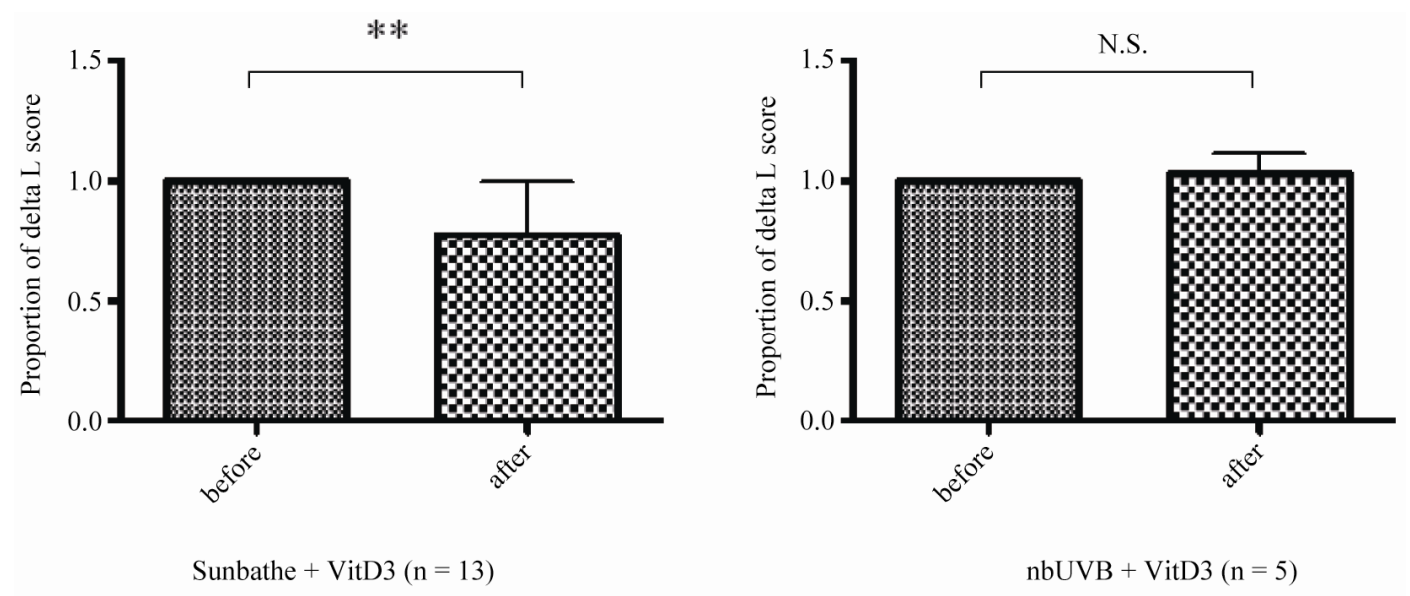

(c)

$$
\text { *: } \mathrm{P}=0.0023
$$

Figure 1. (a) Representative clinical features before and after treatment in group A (upper column) and group B (lower column). Hyperpigmentation on the boarder lesion was partly observed; (b) Proportion of patients with repigmentation. Dense column presents group A and light column presents group B; (c) Comparison of delta $L$ scores before and after treatment. There was significant improvement in the delta $L$ score in group A whereas there was no change in group $B$.

and it required to no need to visit the clinic. Vitamin D3 alone is not clinically efficient for vitiligo irrespective of its ability to upregulate stem cell factor and endothelin-1 in vitro [2]. The combination of vitamin D3 with phototherapy yield improved results $[3,4]$, thus the current study compared natural UV irradiation with narrowband UVB. Group A achieved better improvement in the delta L score, which represents the skin whiteness between the lesional and peri-lesional area, while the repigmentation rate was not significantly different between the two groups. This suggests that natural repigmentation without hyperpigmentation on the peripheral area seems to be obtained with solar illumination. Taken together, solar irradiation along with topical vitamin D3 may be an alternative treatment option for sun-exposed lesions such as those on the face and neck. The optimal timing to apply vitamin D3 analogues, as well as the sun exposure time and dosage should be investigated and clarified in future studies.

\section{Acknowledgements}

We thank Kenju Nishida, Eriko Nobuyoshi for their expert technical assistance in statistical analysis. 


\section{REFERENCES}

[1] D. J. Gawkrodger, A. D. Ormerod, L. Shaw, I. MauriSole, M. E. Whitton, M. J. Watts, et al., "Guideline for the Diagnosis and Management of Vitiligo," British Journal of Dermatology, Vol. 159, No. 5, 2008, pp. 10511076. doi:10.1111/j.1365-2133.2008.08881.x

[2] S. A. Birlea, G. E. Costin and D. A. Norris, "New Insights on Therapy with Vitamin D Analogs Targeting the Intracellular Pathways that Control Repigmentation in Human Vitiligo," Medicinal Research Reviews, Vol. 29, No. 3, 2009, pp. 514-546. doi:10.1002/med.20146

[3] O. Ermis, E. Alpsoy, L. Cetin and E. Yilmaz, "Is the Efficacy of Psoralen Plus Ultraviolet A Therapy for Vitiligo Enhanced by Concurrent Topical Calcipotriol? A Pla-
cebo-Controlled Double-Blind Study," British Journal of Dermatology, Vol. 145, No. 3, 2001, pp. 472-475. doi:10.1046/j.1365-2133.2001.04286.x

[4] P. Kullavanijaya and H. W. Lim, "Topical Calcipotriene and Narrowband Ultraviolet B in the Treatment of Vitiligo," Photodermatology, Photoimmunology \& Photomedicine, Vol. 20, No. 5, 2004, pp. 248-251. doi:10.1111/j.1600-0781.2004.00114.x

[5] I. Katayama, M. Ashida, A. Maeda, K. Eishi, H. Murota, and S. J. Bae, "Open Trial of Topical Tacalcitol $\left[1 \alpha 24(\mathrm{OH})_{2} \mathrm{D}_{3}\right]$ and Solar Irradiation for Vitiligo Vulgaris: Upregulation of c-Kit mRNA by Cultured Melanocytes," European Journal of Dermatology, Vol. 13, No. 4, 2003, pp. 372-376. 